\title{
MRI Denoising Based on Neutrosophic Wiener Filtering
}

\author{
J. Mohan \\ Department of ECE, \\ P.A College of Engineering \& Technology, \\ Pollachi, Tamilnadu, India - 642002. \\ jaimohan12@gmail.com \\ Dr.V. Krishnaveni \\ Department of ECE, \\ PSG College of Technology, \\ Coimbatore, Tamilnadu, India - 641004. \\ vk@ece.psgtech.ac.in
}

\author{
Dr. Yanhui Guo \\ Department of Radiology, \\ University of Michigan, \\ Ann Arbor, MI 48109, USA \\ yanhuig@med.umich.edu \\ Dr. Kanchana Jeganathan, \\ Division of Engineering, \\ King's College London, \\ Strand, London, WC2R 2LS. \\ kanchana.jeganathan@kcl.ac.uk
}

\begin{abstract}
In this paper, a new filtering method is presents to remove Rician noise from magnetic resonance image. This filter is based on Neutrosophic set (NS) approach of wiener filtering. A Neutrosophic Set (NS), a part of neutrosophy theory, studies the origin, nature, and scope of neutralities, as well as their interactions with different ideational spectra. Now, we apply the neutrosophic set into image domain and define some concepts and operators for image denoising. The image is transformed into NS domain, described using three membership sets: True (T), Indeterminacy (I) and False (F). The entropy of the neutrosophic set is defined and employed to measure the indeterminacy. The $\omega$-wiener filtering operation is used on $\mathrm{T}$ and $F$ to decrease the set indeterminacy and remove noise. The experiments have conducted on simulated MR images from Brainweb database with Rician noise added. The visual and the diagnostic quality of the denoised image are well preserved. The performance of this filter is compared with anisotropic diffusion filter (ADF) and unbiased non local mean filter (UNLM).
\end{abstract}

Keywords-component; Denoising, magnetic resonance imaging, neutrosophic set, PSNR, Rician distribution, SSIM, QILV, wiener.

\section{INTRODUCTION}

Magnetic Resonance Imaging (MRI) is a powerful diagnostic technique used in radiology to visualize detailed internal structures of the human body. MRI is primarily used to demonstrate pathological or other physiological alterations of living tissues and is a commonly used form of medical imaging [1]. Despite significant improvements in recent years, MR images often suffer from low signal to noise ratio (SNR) especially in cardiac and brain imaging. The noise in MR images follows Rician distribution [2]. This is problematic for further tasks such as segmentation of important features; classification of images for computer aided diagnostics, three dimensional image reconstruction and image registration. Therefore, noise reduction techniques are of great interest in MR imaging.
Numerous approaches of denoising MR images have been proposed in the literatures [3-12]. In this paper, the neutrosophic set approach (NS) of wiener filtering is proposed to remove the Rician noise in MRI. First the noisy MRI is transformed into the neutrosophic set and the $\omega$ - wiener filtering is employed to reduce the indetermination degree of the image, which is measured by the entropy of the indeterminate subset, after filtering, the noise will be removed. The experiments on simulated MR images (Brainweb database [13]) having different Rician noise levels demonstrate that this approach can perform denoising better.

\section{NeUtrosophic MR IMAGE DENOISING}

Neutrosophy, a branch of philosophy introduced in [14] as a generalization of dialectics, studies the origin, nature and scope of neutralities, as well as their interactions with different ideational spectra.

\section{A. Neutrophic Set}

Let $U$ be a Universe of discourse and a neutrosophic set $A$ is included in $U$. An element $x$ in set $A$ is noted as $x(T, I, F) . T, I$ and $F$ are called the neutrosophic components. The element $x(T, I, F)$ belongs to $A$ in the following way. It is $t \%$ true in the set, $i \%$ indeterminate in the set, and $f \%$ false in the set, where $t$ varies in $T, i$ varies in $I$ and $f$ varies in $F$.

\section{B. Transform the image into neutrosophic set}

Let $U$ be a Universe of discourse and $W$ is a set of $U$, which is composed by bright pixels. A neutrosophic image $P_{N S}$ is characterized by three membership sets $T, I, F$. a pixel $P$ in the image is described as $P(T, I, F)$ and belongs 
to $W$ in the following way: It is $t$ true in the set, $i$ indeterminate in the set, and $f$ false in the set, where $t$ varies in $T, i$ varies in $I$ and $f$ varies in $F$. Then the pixel $P(i, j)$ in the image domain is transformed into the neutrosophic

domain $P_{N S}(i, j)=\{T(i, j), I(i, j), F(i, j)\} . T(i, j), I(i, j)$ and $F(i, j)$ are the probabilities belong to white pixels set, indeterminate set and non white pixels set respectively, which are defined as:

$$
\begin{aligned}
& T(i, j)=\frac{\bar{g}(i, j)-\bar{g}_{\text {min }}}{\bar{g}_{\text {max }}-\bar{g}_{\text {min }}} \\
& \bar{g}(i, j)=\frac{1}{w \times w} \sum_{m=i-w / 2}^{i+w / 2} \sum_{n=j-w / 2}^{j+w / 2} g(m, n) \\
& I(i, j)=\frac{\delta(i, j)-\delta_{\min }}{\delta_{\text {max }}-\delta_{\text {min }}} \\
& \delta(i, j)=a b s(g(i, j)-\bar{g}(i, j)) \\
& F(i, j)=1-T(i, j)
\end{aligned}
$$

where $\bar{g}(i, j)$ is the local mean value of the pixels of the window. $\delta(i, j)$ is the absolute value of difference between intensity $g(i, j)$ and its local mean value $\bar{g}(i, j)$.

\section{Neutrosophic image entropy}

For a gray image, the entropy is utilized to evaluate the distribution of the gray levels. If the entropy is the maximum, the intensities have equal probability. If the entropy is small, the intensity distribution is non-uniform.

Neutrosophic entropy of an image is defined as the summation of the entropies of three subsets $T, I$ and $F$ :

$$
\begin{aligned}
& E n_{N S}=E n_{T}+E n_{I}+E n_{F} \\
& E n_{T}=-\sum_{i=\min \{T\}}^{\max \{T\}} p_{T}(i) \ln p_{T}(i) \\
& E n_{I}=-\sum_{i=\min \{I\}}^{\max \{I\}} p_{I}(i) \ln p_{I}(i) \\
& E n_{F}=-\sum_{i=\min \{F\}}^{\max \{F\}} p_{F}(i) \ln p_{F}(i)
\end{aligned}
$$

where $E n_{T}, E n_{I}$ and $E n_{F}$ are the entropies of sets $T, I$ and $F$ respectively. $p_{T}(i), p_{I}(i)$ and $p_{F}(i)$ are the probabilities of elements in $T, I$ and $F$ respectively, whose values equal to $i$.

\section{D. $\omega$-wiener filtering operation}

The values of $I(i, j)$ is employed to measure the indeterminate degree of element $P_{N S}(i, j)$. To make the set $I$ correlated with $T$ and $F$, the changes in $T$ and $F$ influence the distribution of element in $I$ and vary the entropy of $I$. A $\omega$ - wiener filtering operation for $P_{N S}, \hat{P}_{N S}(\omega)$, is defined as:

$$
\begin{aligned}
& \hat{P}_{N S}(\omega)=P(\hat{T}(\omega), \hat{I}(\omega), \hat{F}(\omega)) \\
& \hat{T}(\omega)= \begin{cases}T & I<\omega \\
\hat{T}_{\omega} & I \geq \omega\end{cases} \\
& \hat{T}_{\omega}(i, j)=\underset{(m, n) \in S_{i, j}}{\text { wiener }_{i}}\{T(m, n)\} \\
& \hat{F}(\omega)= \begin{cases}F & I<\omega \\
\hat{F}_{\omega} & I \geq \omega\end{cases} \\
& \hat{F}_{\omega}(i, j)=\underset{(m, n) \in S_{i, j}}{w i e n e r}\{F(m, n)\} \\
& \hat{I}_{\omega}(i, j)=\frac{\delta_{\hat{T}}(i, j)-\delta_{\hat{T} \min }}{\delta_{\hat{T} \max }-\delta_{\hat{T} \min }} \\
& \delta_{\hat{T}}(i, j)=a_{b s}(\hat{T}(i, j)-\hat{\hat{T}}(i, j))
\end{aligned}
$$

$$
\overline{\hat{T}}(i, j)=\frac{1}{w \times w} \sum_{m=i-w / 2}^{i+w / 2} \sum_{n=j-w / 2}^{j+w / 2} \hat{T}(m, n)
$$

where $\delta_{\hat{T}}(i, j)$ is the absolute value of difference between intensity $\hat{T}(i, j)$ and its local mean value $\overline{\hat{T}}(i, j)$ at $(i, j)$ after $\omega$ - wiener filtering operation.

The MRI denoising method based on neutrosophic set approach of wiener filtering is summarized as below:

Step 1: Transform the image into NS domain;

Step 2: Use $\omega$ - wiener filtering operation on the true subset

$$
T \text { to obtain } T_{\omega}
$$

Step 3: Compute the entropy of the indeterminate subset

$$
\hat{I}_{\omega}, E n_{\hat{I}_{\omega}}(i)
$$

Step 4: if $\frac{E n_{\hat{I}_{\omega}}(i+1)-E n_{\hat{I}_{\omega}}(i)}{E n_{\hat{I}_{\omega}}(i)}<\delta$, go to Step 5;

Else $T=\hat{T}_{\omega}$, go to Step 2;

Step 5: Transform subset $\hat{T}_{\omega}$ from the neutrosophic domain into the gray level domain. 


\section{RESULTS AND DISCUSSION}

The experiments are conducted on simulated MR images obtained from the Brainweb database [13]. The dataset consists of T1 and T2- weighted MR images. The experiments are performed on various images (T1 and T2- weighted normal MR images; T1 and T2- weighted MR images with Multiple Sclerosis) degraded by different noise levels. Here, the performance of the proposed method is demonstrated for T1-weighted MR image of normal brain with $9 \%$ noise level and T1-weighted brain MR image with MS lesion corrupted by $9 \%$ noise level. This method is compared with Anisotropic Diffusion Filter (ADF) [3] and Unbiased Non Local Mean (UNLM) filter [11]. The results of the different denoising filters on a T1-weighted MR image of normal brain and with MS lesion corrupted by $9 \%$ Rician noise level are shown in Fig. 1 and Fig. 2 respectively. From these results, the proposed denoising filter produce good denoised image in terms of visual perception.

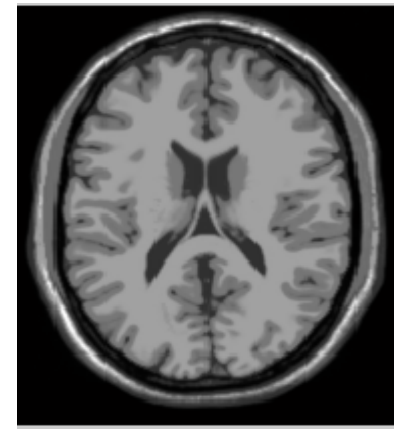

(a)

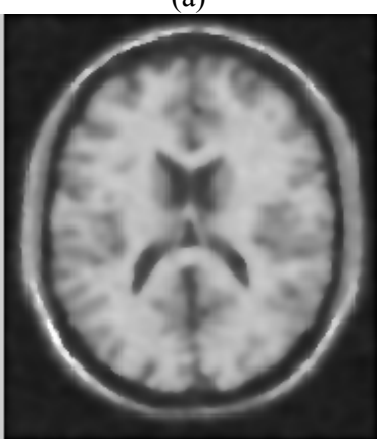

(c)

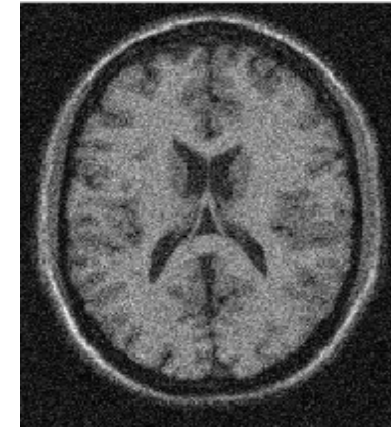

(b)

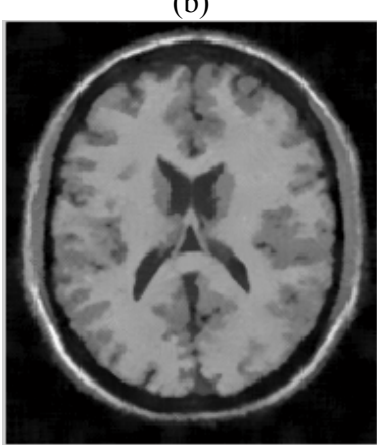

(d)

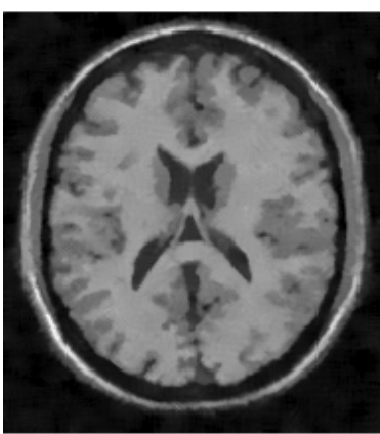

(e)

Figure 1. Results of different denoising filters on a T1-weighted MR image of normal brain with $9 \%$ noise level. From top to bottom: (a) the original image, (b) the noise corrupted image, denoised images using (c) AD filter, (d) UNLM filter and (e) NS wiener filter.

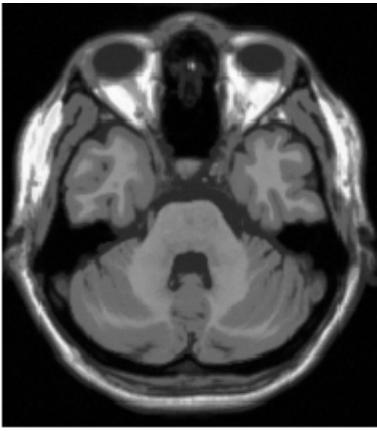

(a)

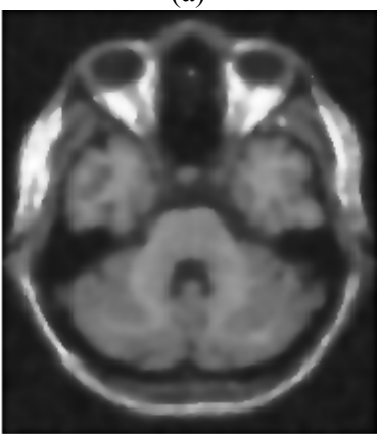

(c)

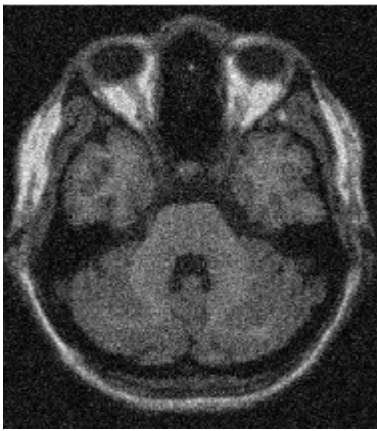

(b)

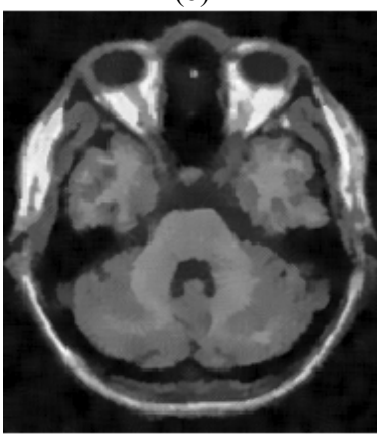

(d)

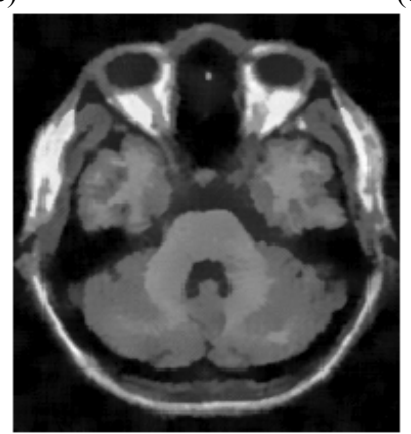

(e)

Figure 2. Results of different denoising filters on a T1-weighted MR image of MS lesion brain with 9\% noise level. From top to bottom: (a) the original image, (b) the noise corrupted image, denoised images using (c) AD filter, (d) UNLM filter and (e) NS wiener filter

The performance of the denoising algorithm is measured by using the quality metrics such as peak-signal-to-noise ratio (PSNR), the Structural Similarity (SSIM) index [15] and the Quality Index based on Local Variance (QILV) [16]. The peak signal to noise ratio in decibel $(\mathrm{dB})$ is measured using the following formula:

$$
P S N R=-10 \log \left[\frac{\sum_{i=0}^{i=H-1} \sum_{j=0}^{j=W-1}\left(I(i, j)-I_{d}(i, j)\right)^{2}}{H \times W \times 255^{2}}\right]
$$

where $I(i, j)$ and $I_{d}(i, j)$ represent the intensities of pixels $(i, j)$ in the original image and denoised image respectively. The higher the PSNR, the better the denoising algorithm is. SSIM and QILV give the measure of the structural similarity between the original and the denoised images and are in the range of 0 to 1 . The SSIM works as follows: Let $x$ and $y$ be 
two non negative images, where as one has perfect quality. Then, the SSIM can serve as a quantitative measure of the similarity of the second image. The system separates the task of similarity measurement into three comparisons: luminance, contrast and structure. It can be defined as

$$
\operatorname{SSIM}(x, y)=\frac{\left(2 \mu_{x} \mu_{y}+C_{1}\right)\left(2 \sigma_{x y}+C_{2}\right)}{\left(\mu_{x}^{2}+\mu_{y}^{2}+C_{1}\right)\left(\sigma_{x}^{2}+\sigma_{y}^{2}+C_{2}\right)}
$$

where $\mu_{x}$ and $\mu_{y}$ are the estimated mean intensity and $\sigma_{x}$ and $\sigma_{y}$ are the standard deviations respectively. $\sigma_{x y}$ can be estimated as

$$
\sigma_{x y}=\frac{1}{N-1} \sum_{i=1}^{N}\left(x_{i}-\mu_{x}\right)\left(y_{i}-\mu_{y}\right)
$$

$C_{1}$ and $C_{2}$ in eqn. 19 are constants and the values are given as $C_{1}=\left(K_{1} L\right)^{2}$ and $C_{2}=\left(K_{2} L\right)^{2}$ where $K_{1}, K_{2}<<1$ is a small constant and $L$ is the dynamic range of the pixel values (255 for 8 bit gray scale images).

The QILV between two images $x$ and $y$ can be defined as

$$
\operatorname{QILV}(x, y)=\frac{2 \mu_{V x} \mu_{V y}}{\mu_{V x}^{2}+\mu_{V y}^{2}} \cdot \frac{2 \sigma_{V x} \sigma_{V y}}{\sigma_{V x}^{2}+\sigma_{V y}^{2}} \cdot \frac{\sigma_{V x V y}}{\sigma_{V x} \sigma_{V y}}
$$

where $\mu_{V x}$ and $\mu_{V y}$ are the estimated means of the local variance and $\sigma_{V x}$ and $\sigma_{V y}$ are the standard deviations of the local variance respectively. $\sigma_{V x V y}$ is the covariance between the two images.

The efficiency of the proposed denoising filter is compared with ADF and UNLM. The experiments carried on a simulated T1-weighted MR image of normal brain with $1 \%, 3 \%, 5 \%$, $7 \%, 9 \%, 15 \%$ and $21 \%$ of Rician noise levels are added. The quality metrics PSNR, SSIM and QILV are calculated and the comparison of these metrics for ADF, UNLM and proposed filter is given in Fig. 3 and Fig. 4. The PSNR, SSIM and QILV values obtained for the T1 and T2 weighted images using the aforementioned denoising techniques are tabulated in Table I. From these results, the proposed filter provides better denoising by obtaining high PSNR, High SSIM and high QILV for all the values of noise levels.

TABLE I

Comparison of the Denoising TeChNiques Based on the Performance METRICS FOR DIFFERENT MR IMAGES

\begin{tabular}{|l|l|c|c|c|}
\hline \multirow{2}{*}{ MR Image } & \multirow{2}{*}{$\begin{array}{l}\text { Denoising } \\
\text { Methods }\end{array}$} & \multicolumn{3}{|l|}{ Performance Metrics } \\
\cline { 3 - 5 } & & PSNR(dB) & SSIM & QILV \\
\hline \multirow{2}{*}{$\begin{array}{l}\text { T1- Weighted brain MRI } \\
\text { corrupted by 7\% Rician } \\
\text { noise }\end{array}$} & ADF & 23.98 & 0.962 & 0.995 \\
\cline { 2 - 5 } & UNLM & 29.72 & 0.9908 & 0.7309 \\
\cline { 2 - 5 } & NS Wiener & $\mathbf{3 1 . 9 1}$ & $\mathbf{0 . 9 9 4 3}$ & $\mathbf{0 . 9 8 7 1}$ \\
\hline $\begin{array}{l}\text { T1- Weighted brain MRI } \\
\text { with MS lesion corrupted } \\
\text { by 9\% Rician noise }\end{array}$ & ADF & 21.59 & 0.9331 & 0.7702 \\
\cline { 2 - 5 } & UNLM & 24.59 & 0.9714 & 0.9692 \\
\cline { 2 - 5 } & NS Wiener & $\mathbf{2 9 . 9 5}$ & $\mathbf{0 . 9 9 0 3}$ & $\mathbf{0 . 9 9 3}$ \\
\hline \multirow{2}{*}{$\begin{array}{l}\text { T2- Weighted brain MRI } \\
\text { corrupted by 15\% Rician } \\
\text { noise }\end{array}$} & ADF & 18.24 & 0.8808 & 0.6555 \\
\cline { 2 - 5 } & UNLM & 23.44 & 0.9532 & 0.9441 \\
\cline { 2 - 5 } & NS Wiener & $\mathbf{2 4 . 0 8}$ & $\mathbf{0 . 9 6 8 2}$ & $\mathbf{0 . 9 8 8 2}$ \\
\hline
\end{tabular}
and NS Wiener Filters based on the quality measures for T1- weighted normal brain MRI: (a) PSNR (b) SSIM (c) QILV.

\section{CONCLUSION}

In this article, a novel MRI denoising technique based on neutrosophic set approach of wiener filtering has been proposed. The image is described as a NS set using three membership sets $T, I$ and $F$. The entropy in neutrosophic image domain is defined and employed to measure the indetermination. The wiener filter is applied to reduce the set's indetermination and remove the noise in the MR image. The performance of the proposed denoising filter is compared with ADF and UNLM based on PSNR, SSIM and QILV. 


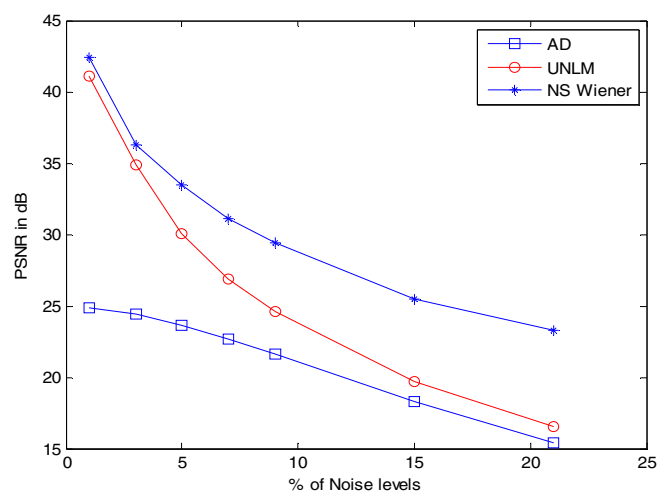

(a)

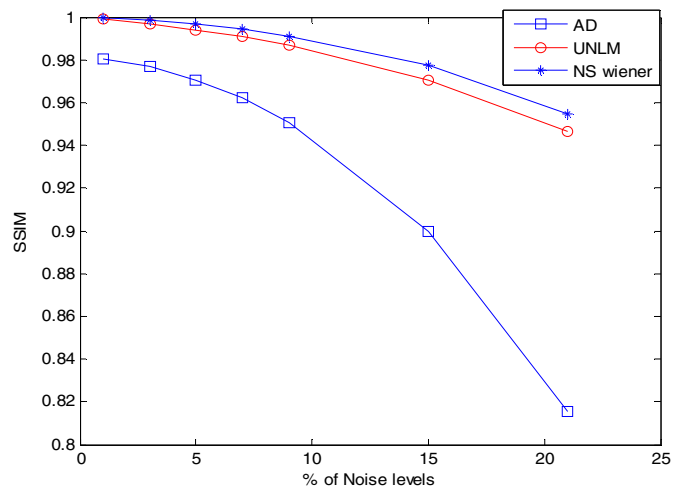

(b)

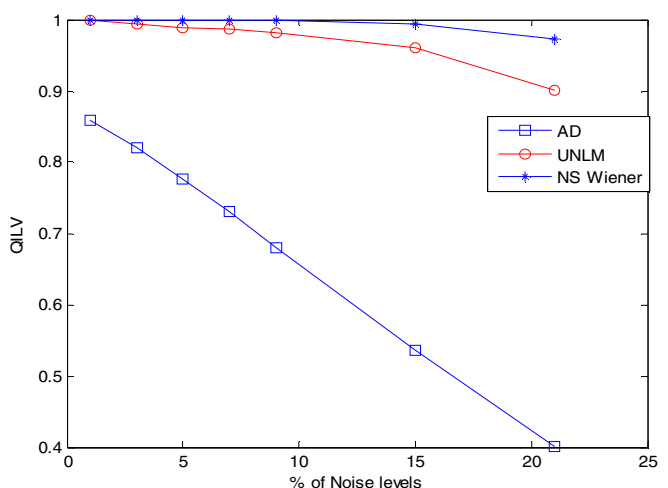

(c)

Figure 4. Comparison of the denoising results obtained from ADF, UNLM, and NS Wiener Filters based on the quality measures for T1- weighted brain MRI with MS lesion: (a) PSNR (b) SSIM (c) QILV

The experimental results demonstrate that the proposed approach can remove noise automatically and effectively. This filtering method tends to produce good denoised image not only in terms of visual perception but also in terms of the quality metrics.

\section{REFERENCES}

[1] G. Wright, "Magnetic Resonance Imaging", IEEE Signal Process Mag. Vol. 1, pp. 56-66, 1997.

[2] H. Gudbjartsson and S. Patz, "The Rician distribution of noisy MRI data," Magn. Reson. Med., vol. 34, no. 6, pp. 910-914, Dec. 1995.
[3] G. Gerig, O. Kubler, R. Kikinis, and F. A. Jolesz, "Nonlinear anisotropic filtering of MRI data," IEEE Trans. Med. Imag., vol. 11, no. 2, pp. 221232, Jun. 1992.

[4] J. Sijbers, A. J. den Dekker, A. Van Der Linden, M. Verhoye and D. Van Dyck, "Adaptive Anisotropic noise filtering for Magnitude MR data”, Magn. Reson. Imaging, vol. 17, no. 10, pp. 1533-1539, Dec. 1999.

[5] K. Krissian and S. Aja-Fernández, "Noise driven Anisotropic Diffusion filtering of MRI", IEEE Trans. Image Processing, vol. 18, no. 10, pp. 2265-2274, Oct. 2009.

[6] R. D. Nowak, "Wavelet-based Rician noise removal for magnetic resonance imaging," IEEE Trans. Image Process., vol. 8, no. 10, pp. 1408-1419, Oct. 1999.

[7] A. Pizurica, W. Philips, I. Lemahieu, and M. Acheroy, "A versatile wavelet domain noise filtration technique for medical imaging," IEEE Trans. Med. Imag., vol. 22, no. 3, pp. 323-331, Mar. 2003.

[8] P. Bao and L. Zhang, "Noise reduction for magnetic resonance images via adaptive multiscale products thresholding," IEEE Trans. Med. Imag., vol. 22, no. 9, pp. 1089-1099, Sep. 2003.

[9] A. Buades, B. Coll, and J. M. Morel, "A review of image denoising algorithms, with a new one," Multiscale Modeling and Simulation, vol. 4, pp. 490-530, 2005.

[10] P. Coupe, P. Yger, S. Prima, P. Hellier, C. Kervrann, and C. Barillot, "An optimized blockwise nonlocal means denoising filter for 3-D magnetic resonance images," IEEE Trans. Med. Imag., vol. 27, no. 4, pp. 425-441, Apr. 2008.

[11] J. V. Manjón, J. Carbonell-Caballero, J. J. Lull, G. García-Martí, L. Martí-Bonmatí, and M. Robles, "MRI denoising using non-local means," Med. Image Anal., vol. 12, no. 4, pp. 514-523, Aug. 2008.

[12] J. V. Manjón, P. Coupe, L. Martí-Bonmatí, D. L. Collins and M. Robles, "Adaptive Non-Local Means Denoising of MR images with spatially varying noise levels", Magn. Reson. Imaging, vol. 31, no. 1, pp. 192203, Jan. 2010.

[13] R. K. Kwan, A. C. Evans, G. B. Pike, "MRI simulation based evaluation of image processing and classification methods," IEEE trans. Med. Imag., vol. 18, no. 11, pp. 1085-1097, 1999.

[14] F. Samarandache, A unifying field in logics Neutrosophic logic, in Neutrosophy. Neutrosophic Set, Neutrosophic Probability, $3^{\text {rd }}$ edition, American Research Press, 2003.

[15] Z. Wang, A. C. Bovik H.R. Sheikh, E.P. Simoncell. "Image quality assessment:From error visibility to structural similarity", IEEE Trans. Image Process, vol. 13, pp. 600-612, 2004.

[16] S. Aja-Fernandez, R. San-Jose-Estepar, C. Alberola-Lopaz, C.F.Westin. "Image quality assessment based on local variance". in Proceedings $28^{\text {th }}$ IEEE EMBC, New York pp.4815-4818, 2006. 